\title{
VARIAÇĀO SAZONAL NA PREVALÊNCIA DE LEPTOSPIROSE EM CÃES DE RUA DA CIDADE DE SÃO PAULO, BRASIL *
}

\author{
P. H. Yasuda ** \\ C. A. Santa Rosa \\ R. M. Yanaguita **
}

RSPUB9/533

\begin{abstract}
YASUdA, P. H. et al. Variação sazonal na prevalência de leptospirose em cães de rua da cidade de São Paulo, Brasil. Rev. Saúde públ., S. Paulo, 14:
\end{abstract} $589-96,1980$.

RESUMO: Utilizando a tecnica da soroaglutinaçāo microscópica para o diagnóstico de leptospirose, $308(21,6 \%)$ de 1428 soros de cáes errantes da cidade de São Paulo (Brasil) mostraram-se reagentes. $\mathrm{Na}$ população canina estudada, a infeccão leptospirótica sofreu influência sazonal. Verâo $(24,2 \%)$ e outono (24,9\%) foram as estaçóes do ano com maior número de soros reatores, em oposiçáo a primavera $(18,3 \%)$ e inverno $(18,3 \%)$. Estas diferenças foram significantes, estatisticamente. O sorotipo canicola é o principal causador da leptospirose na população estudada $(50,7 \%)$, seguido do icterohaemorrhagiae $(25,5 \%)$; grippotyphosa $(7,8 \%)$; pomona $(6,7 \%)$ e ballum $(4,4 \%)$.

Unitermos: Leptospirose, S. Paulo, SP, Brasil. Cães. Soroaglutinação. Estaf̧ão do ano.

\section{INTRODUÇAO}

O papel do cão na epidemiologia da leptospirose tem sido destacado nos últimos anos 6.14 . A população canina na cidade de São Paulo está estimada em cerca de 820.000 animais, sendo muitos deles errantes. Os dados existentes em nosso meio, com relação à leptospirose em cães são insuficientes e, além disso, nunca se analisaram aspectos como a variação sazonal na prevalência da infecção por leptospiras $5,8,9,12,17$.
Devido ao crescimento desordenado da cidade de São Paulo, muitos de seus bairros apresentam problemas de saneamento básico e condições ecológicas favoráveis à proliferação de roedores, que são, em nosso meio, os grandes portadores do microrganismo em questão. O uso comum do mẹic ambiente com tais roedores tornam 0 homem, o cão, assim como outros animais domésticos, suscetiveis de se contaminarem e de propagarem entre si a leptospirose.

* Apresentado no X Congresso Brasileiro de Microbiologia, Rlo de Janeiro, 1979.

* Do Departamento de Microbiologia e Imunologia do Instituto de Ciēncias Biomédicas da USP - Setor Medicina - Av. Dr. Arnaldo, 455 - 01246 - São Paulo, SP - Brasil.

*.. Do Departamento de Medicina Preventiva e Saúde Animal da Faculdade de Medicina Veterinária e Zootecnica da USP - Cidade Universitária Armando Sales de Oliveira - 05568 São Paulo, SP - Brasil. 
YASUDA, P. H. et al. Variação sazonal na prevalência de leptospirose em cães de rua da cidacio de São Paulo, Brasil. Rev. Saúde públ., S. Paulo, 14:589-96, 1980.

Com a presente comunicação, espera-se esclarecer alguns aspectos epidemiológicos desta importante zoonose, ressaltando-se as variações ocorridas na prevalência de infecção em cães ao longo de um ann.

\section{MATERIAL E METODOS}

Um total de 1428 soros de cães de rua foi colhido no periodo compreendido entre outubro de 1976 a setembro de 1977.

Os cães procediam de capturas diárias feitas em 15 Regionais Administrativas de que está constituida a cidade de São Paulo (Fig. 1) e que foram reagrupadas da seguinte maneira:

São Miguel-E. Matarazzo e Itaquera-Guaianazes;

Penha e Santana;

Freguesia do $O$ e Pirituba-Perús;
Lapa, Pinheiros e Butantã;

Campo Limpo e Santo Amaro;

Vila Mariana e Ipiranga;

Moóca e Vila Prudente.

A técnica sorológica empregada foi a de aglutinação microscópica ${ }^{15}$, utilizando-se como antigenos os seguintes sorotipos de leptospiras: icterohaemorrhagiae, canicola, pomona, grippotyphosa, tarassovi, australis, bataviae, ballum, wolffi, panama, pyrogenes, javanica, autumnalis, butembo, andamana, shermani, whitcombi e brasiliensis.

Foram considerados soros positivos aqueles que apresentaram aglutinação na diluição igual ou superior a 1:100.

Os dados obtidos foram submetidos a análise estatística, utilizando-se o teste do Qui-quadrado. Em todos os casos adotouse o nivel de significância de $0,05 \quad(5 \%)$ para a exclusão da hipótese.

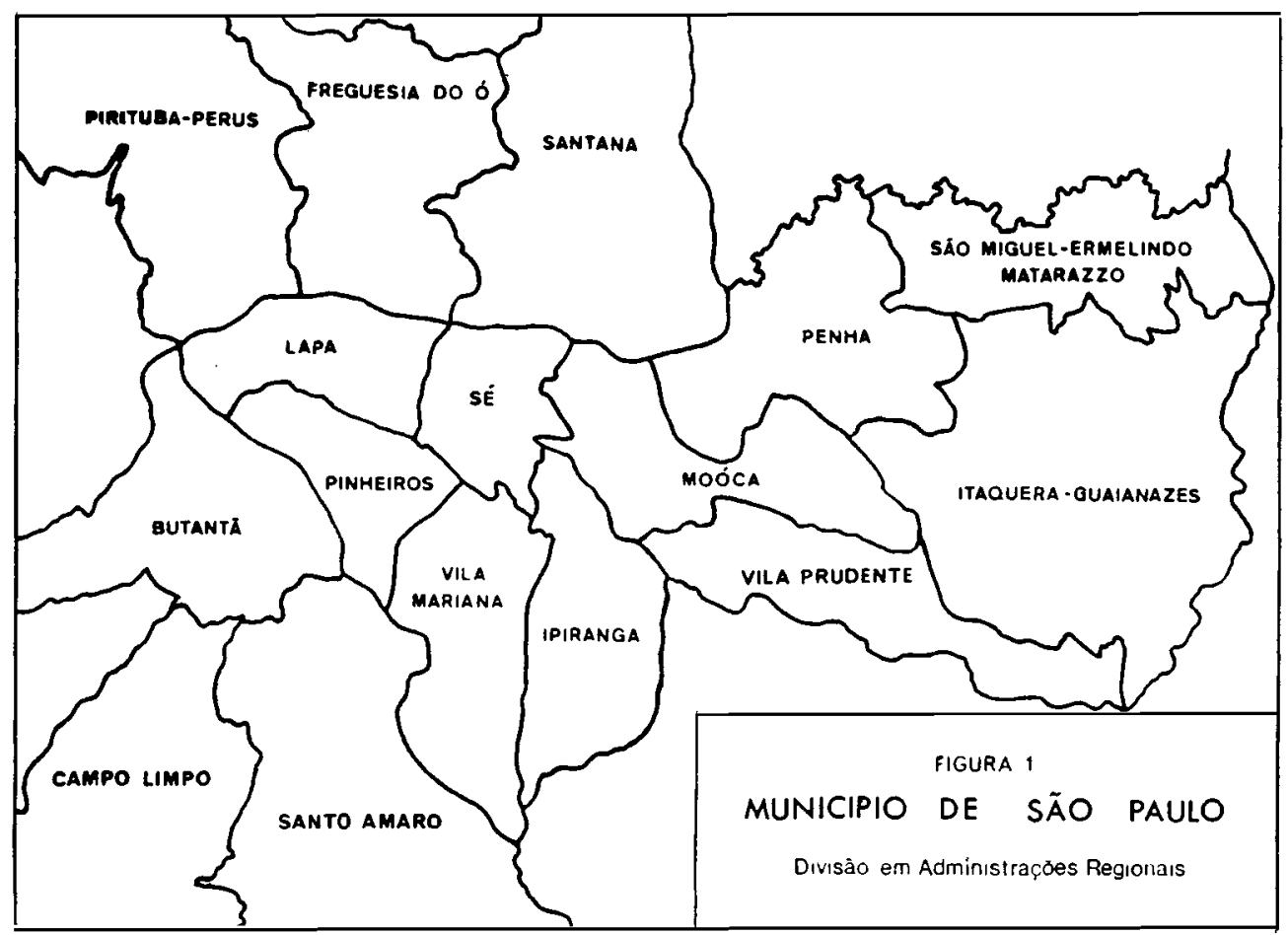

Fig. 1 - Municipio de São Paulo, Divisão em Administrações Regionais. 
YASUDA, P. H. et al. Variação sazonal na prevalência de leptospirose em cảes de rua da cidade de São Paulo, Brasil. Rev. Saúde pübl., S. Paulo, 14:589-96, 1980.

\section{RESULTADOS}

Dos 1.428 soros de cães submetidos à aglutinação microscópica, $308(21,6 \%)$ foram reagentes a um ou mais sorotipos de leptospiras.

\section{Varią̧äo mensal}

A variação mensal na prevalência de soros reagentes para leptospirose está apresentada na Tabela 1. Essas variações submetidas à análise estatística, não se mostraram significantes.

T A B EL A 1

Frequîencia mensal de soros com aglutininas antileptospiras (título $\geqslant 100$ ) em cães de rua da cidade de São Paulo, período de outubro/76 a setembro/77.

\begin{tabular}{|c|c|c|}
\hline \multirow{2}{*}{ Meses } & ne de soros positivos & \multirow{2}{*}{$\begin{array}{l}\text { Percentagem } \\
\text { de positividade }\end{array}$} \\
\hline & n* de soros examinados & \\
\hline Outubro & $17 / 92$ & 18,5 \\
\hline Novembro & $16 / 102$ & 15,7 \\
\hline Dezembro & $22 / 106$ & 20.7 \\
\hline Janeiro & $20 / 112$ & 17,8 \\
\hline Fevereiro & $27 / 102$ & 26.5 \\
\hline Março & $40 / 148$ & 27,0 \\
\hline Abril & $40 / 139$ & 28,8 \\
\hline Maío & $37 / 159$ & 23,3 \\
\hline Junho & $27 / 140$ & 19,3 \\
\hline Julho & $27 / 123$ & 21.9 \\
\hline Agosto & $15 / 107$ & 14,0 \\
\hline Setembro & $20 / 98$ & 20,4 \\
\hline Total & $308 / 1428$ & 21,6 \\
\hline
\end{tabular}

\section{Variasão sazonal}

As freqüências, por estações do ano, de soros com aglutininas antileptospiras em cães estudados estão apresentadas na Tabela 2. As diferenças na prevalência de leptospirose ocorridas no verão e outono foram estatisticamente significantes das ocorridas na primavera e inverno. O método estatístico empregado neste caso foi $o$ de Grizzle e col.:

\section{Variaçīo por Regionais}

As frequiências de soros com aglutininas antileptospiras nos cāes distribuídas por Regionais estão apresentadas na Tabela 3. Em termos estatísticos, as diferenças ocorridas não foram significantes.
$\mathrm{Na}$ Tabela 4 estão apresentadas as freqüèncias de soros positivos observadas nas Regionais durante 0 períndo sazonal estudado.

\section{Caracterização dos soros positivos}

Considerou-se, no presente estudo, o soro positivo correspondente ao sorotipo de leptospira com o qual se obteve o maior título, embora este critério não seja o mais adequado:

De 308 soros reagentes para leptospirose, 38 deles coaglutinaram dois ou mais sorotipos de leptospiras com títulos máximos coincidentes e, portanto, foram eliminados das considerações que serão feitas a seguir. 
YASUDA, P. H, et al. Variação sazonal na prevaléncia de leptospirose em cães de rua da cidade de São Paulo, Brasil. Rev. Saúde pübl., S. Paulo, 14:589-96, 1980.

\section{T A B E L A 2}

Frequència de soros com aglutininas antileptospiras (título $\geqslant 100$ ) em cães de rua da cidade de $\mathbf{S a n o ~ P a u l o , ~ s e g u n d o ~ a s ~ e s t a c ̧ o ̄ e s ~ d o ~ a n o , ~ n o ~ p e r i o d o ~ d e ~}$ outubro/76 a setembro/77.

\begin{tabular}{|c|c|c|}
\hline \multirow[t]{2}{*}{ Estaçōes } & ny de soros positivos & \multirow{2}{*}{$\begin{array}{l}\text { Percentagem } \\
\text { de positividade }\end{array}$} \\
\hline & $\mathbf{n}^{\varphi}$ de soros examinados & \\
\hline Primavera & $55 / 300$ & 18.3 \\
\hline Verāo & $79 / 326$ & 24,2 \\
\hline Outono & $102 / 409$ & 24,9 \\
\hline Inverno & $72 / 393$ & 18,3 \\
\hline Trital & $308 / 1428$ & 21,6 \\
\hline
\end{tabular}

TABEL A

Frequência de soros com aglutininas antileptospiras (título $\geqslant 100$ ) em cães de rua da cidade de São Paulo. segundo as Regionais, no período de outubro/76 a setembro/77.

\begin{tabular}{|c|c|c|}
\hline Regionais & $\begin{array}{l}\text { ne de sotos positivos } \\
\text { Ho de soros examinados }\end{array}$ & $\begin{array}{l}\text { Percentagem } \\
\text { de positividade }\end{array}$ \\
\hline Itaquera-Guaianazes e & & \\
\hline S. Miguel-E. Matarazzo & $50 / 206$ & 24,3 \\
\hline Penhit e Santana & $45 / 203$ & 22.2 \\
\hline $\begin{array}{l}\text { Freguesia do o a } \\
\text { Pirituba-Perús }\end{array}$ & $50 / 206$ & 24.3 \\
\hline Lapa, Pinheiros e Butantã & $36 / 204$ & 17,6 \\
\hline $\begin{array}{l}\text { Campo Limpo e Santo } \\
\text { Amaro }\end{array}$ & $43 / 208$ & 20,7 \\
\hline Vila Mariana $a$ Ipiranga & $42 / 195$ & 21,2 \\
\hline Móca e Vila Prudente & $42 / 208$ & 20,7 \\
\hline Total & $308 / 1428$ & 21.6 \\
\hline
\end{tabular}


YASUDA. P. H. et al. Variação sazonal na prevaléncia de leptospirose em cães de rua da cidade de São Paulo, Brasil. Rev. Saúde públ., S. Paulo. 14:589-96. 1980.

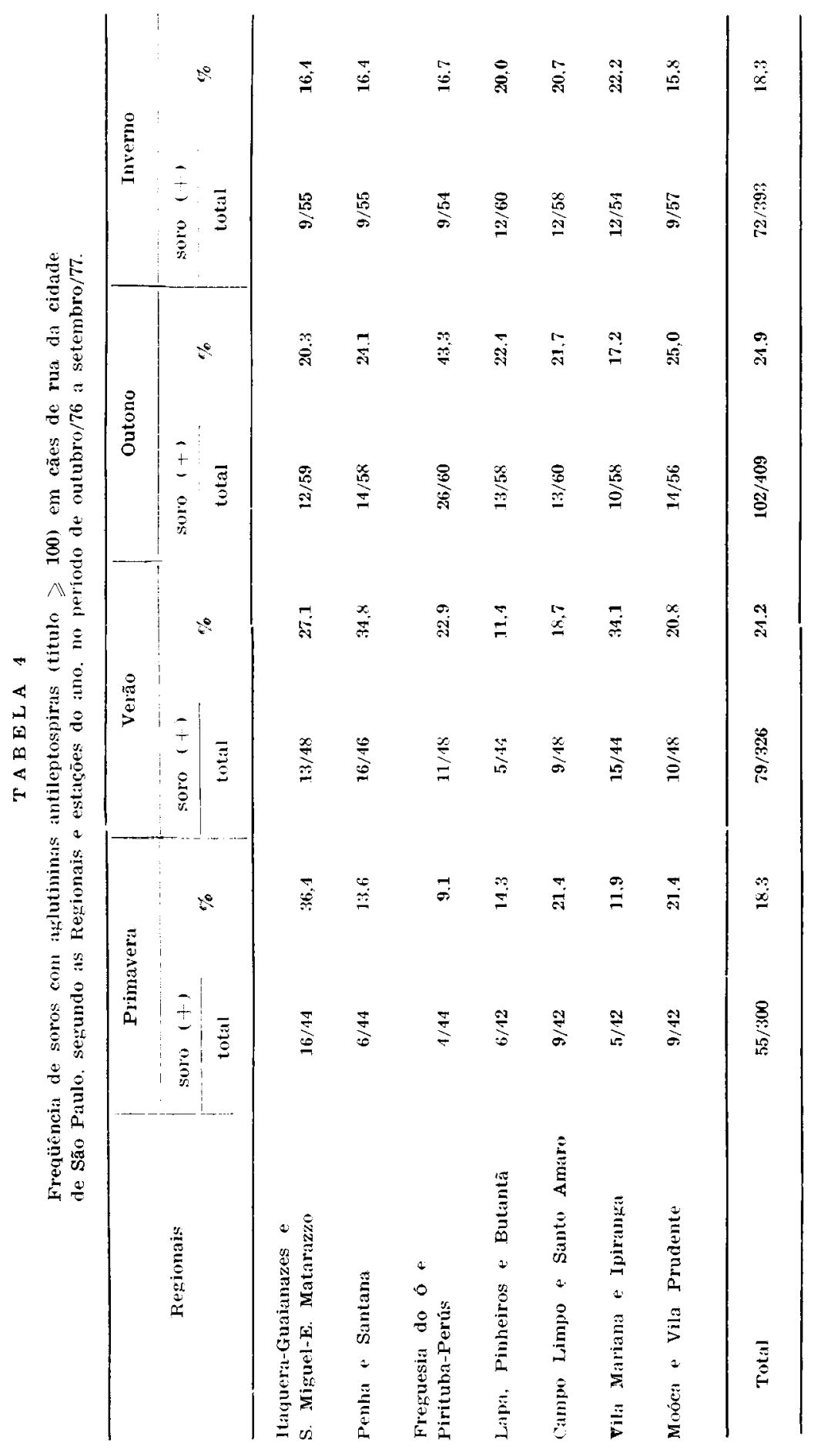


YASUDA, P. H. et al. Variação sazonal na prevalência de leptospirose em cães de rua da cida ̃e de São Paulo. Brasil. Rev. Saúde puibl., S. Paulo, 14:589-96, 1980.

\section{T A B F L A 5}

Distribuição de 270 soros de cães de rua da cidade de São Paulo com aglutininas antileptospiras (título $\geqslant 100$ ), segundo os sorotipos para os quais foram reagentes e as estaçōes do ano, no período de outubro/76 a setembro/77.

\begin{tabular}{l|c|ccc|c}
\hline \multirow{2}{*}{ Sorotipos } & Primaveri & Verão & Outono & Inverno & Total \\
\cline { 2 - 6 } canicola & 21 & 45 & 47 & 24 & 137 \\
icterohaemorrhagiae & 21 & 9 & 16 & 23 & 69 \\
grippotyphosa & 0 & 5 & 9 & 7 & 21 \\
pomona & 3 & 4 & 8 & 3 & 18 \\
ballum & 1 & 2 & 7 & 2 & 12 \\
outros * & 3 & 2 & 4 & 4 & 13 \\
\hline Total & 49 & 67 & 91 & 63 & $270^{* *}$ \\
\hline * outros = pyrogenes, autumnalis, andamana e butembo. & & \\
** devido ao fenomeno de coaglutinação, 38 soros não constam desta Tabela.
\end{tabular}

Assim, em 270 dos 308 soros positivos para leptospirose foi definido o sorotipo responsável pela infecção. Conforme a ilustração da Fig. 2, observa-se que $50,7 \%$ (137 soros) foram reagentes para o sorotipo canicola; $25,5 \%$ (69 soros) para o sorotipo icterohaemorrhagiae e, seguindo-se em ordem decrescente: grippotyphosa, 7,8\% (21 soros); pomona, 6,7\% (18 soros); ballum, $4,4 \%$ (12 soros); pyrogenes, $1,8 \%$ (5 soros); autumnalis, 1,8\% (5 soros); andamana, $0,7 \%$ (2 soros) e finalmente, butembo, $0,4 \%$ (1 soro).

\section{Freqüência dos sorotipos infectantes segundo as estações do ano.}

Pela Tabela 5, observa-se que a maior prevalência de soros reagentes ao sorotipo canicola ocorreu nos meses correspondentes ao verão e outono, 45 e 47 casos, respectivamente. Soros reagentes contra o sorotipo icterohaemorrhagiae foram mais freqüentes nos meses da primavera e do inverno.

\section{DISCUSSAOO}

Uma das características mais interessantes na frequêencia da leptospirose é o selı caráter sazonal. No Brasil, pais onde predomina o clima tropical e sub-tropical em grande parte do seu território, seria de se

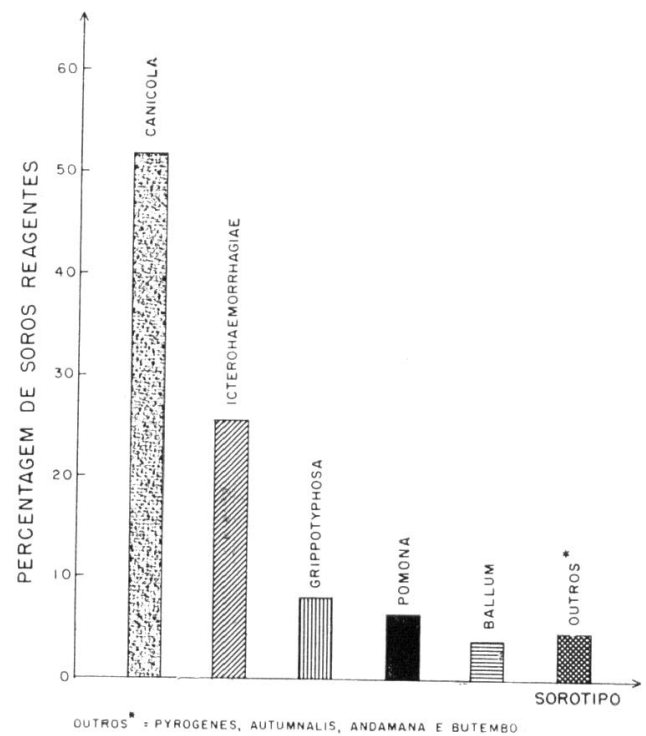

Fig. 2 - Frequência de soroaglutininas, antileptospiras (título $\geqslant 100$ ) de 270 cães de rua da cidade de Såo Paulo, segundo os sorotipos para os quais foram reagentes, no periodo de outubro/76 a setembro/77. 
YASUDA, P. H. et al. Variação sazonal na prevalência de leptospirose em cães de rua da cidade de Săo Paulo, Brasil, Rev. Saúde públ., S. Paulo, 14:589-96, 1980.

esperar que os fatores climáticos também exercessem influência nesta importante zoonose. Embora estes fatos sejam conhecidos para outras regiōes, pouco se sabe a respeito em nosso meio $1,2,4,10,11,13$.

Levantamentos soroepidemiológicos anteriormente efetuados em cães na cidade de São Paulo não oferecen uma visão muito clara a respeito dos sorotipos mais prevalentes na população canina, assinı como as freqüências relatadas pelos diferentes autores variam dentro de uma faixa muito anpla $i, x, y, 12,17$.

Pelos resultados obtidos na presente investigação, observa-se que a leptospirose canina en São Paulo sofre influencia sazonal; verão e outono são periodos onde se cncontram maior número de (ães com sorologia positiva. Tal fato se deve, provavelmente, à ocorrència de chuvas abundantes nas épocas de verāo, facilitando a maior propagação e permanência de leptospiras viáveis fora de um hospedeiro ${ }^{16}$. Nos me- ses de outono, as temperaturas amenas e a clevada umidade do ar, também favoreciam a manutenção de leptospiras no meio anbiente.

Estas observaçōes parecem estar de acordo quando se trata da infecção pelo surotipo canicola. O mesmo não ocorreu con " icterohaemorrhagiae pois, justamente nos meses correspondentes ao verão observa-se un menor numero de soros reatores para este sorotipo (Tabela 5).

Esperava-se encontrar uma distribuiçár, não homogênea da leptospirose em cães por Regionais, visto que as que estão situadas mais na periferia da cidade apresentam problemas graves de saneamento, con deficiências nas redes de esgoto e de água encanada, ruas sem pavimentação, moradias inadequadas e presença de inúmeros terrenos baldios cobertos, geralmente, por vegetação abundante, a qual propicia a multiplicação de ratos, os grandes portadores de leptospiras em nosso meio.

YASUDA, P. H. et al. /Seasonal variation in the prevalence of leptospirosis in stray dogs in the city of S. Paulo (Brazil)/. Rev. Saúde públ., S. Paulo, 14: 589-96, 1980 .

ABSTRACT: One thousand, four hundred and twenty-eight sera from stray dogs from the city of $S$. Paulo were examined using agglutination microscopic testing. Of the total of 1428 sera, $308(21.6 \%)$ showed positive. Seasonal influence was observed in the leptospiral infection of the canine population studied in that the largest number of the reagent sera was verified in the summer (24.2\%) and autumn $(24.9 \%)$. In contrast, spring $(18.3 \%)$ and winter $(18.3 \%)$ prevalence levels were lower. These differences were considered significant from the statistical point of view. The canicola serotype was found to be the main cause of leptospirosis in the population examined $(50.7 \%)$, followed by icterohaemorrhagiae $(25.5 \%)$, grippetyphosa $(7.8 \%)$, pomona $(6.7 \%)$, and ballum $(4.4 \%)$.

Uniterms: Leptospirosis, S. Paulo, SP, Brazil. Dogs. Agglutination tests. Seasons. 
YASUDA, P. H. et al. Variação sazonal na prevalència de leptospirose em cães de rua du cidade de São Paulo, Brasil. Rev. Saúde mibl., S. Paulo. 14:589-96, 1980.

\section{IREFERENCIAS BIBLIOGRAFICAS}

1. Alston, J. M. \& BROOM, J. C. Leptospirosis in man and animals. Edinburgh. Livingstone, 1958.

2. AUSToni. M. Le letospirosi. Padora, Tipografia del Seminario, 1953.

3. BABUDIERI, B. Recerche serolugiche sulla diffusione delle leptospirosi nella provincia di Siviglia. Rend. Ist. Sup. Sanitd, 23:515-23, 1960.

4. BORG-PETERSEN, C. Experience of leptospirosis in Denmark. Proc. roy. Soc. Med., $42: 714-8,1949$.

5. CASTro, A. F. P. de et al. Leptospirose canina em São Paulo. Inquérito sorológico e isolamento da $L$. icterohaemorrhagiae. Arq. Inst. biol, São Paulo, $29: 199-205,1962$.

6. FELDMANN, B. M. The problem of urban dog. Science, $185: 903,1974$.

7. GRIZZLE, J, E. et al. Analysis of categorical data by linear models. Biometrics, 25:489-504, 1969.

8. GUIDA, V. O. Estudos sobre a leptospirose canina. III - Presença de aglutininas - lisinas em soro de cães da cidade de São Paulo. Rco. bras. Biol., 9;35-7, 1949.

9. GUIDA, V. O. \& MONICI, N. Incidência de leptospiras em diversas espécies de animais. II - Frequeuência no cão ( $\mathrm{Ca}$ nis familiaris). Rev. paul. Med., 35: 425-8, 1949.
10. HERRER, A. et al. Leptospirosis en el Peru. I - Identificacion de las cepas de Ieptospiras presentes en el perro $y$ el gato e incidencia de la infeccion. Rev. Med. exp., Lima, 12:65-86, 1958.

11. JOSHUA, J, O. Discussion on leptospirosis. Proc. roy. Soc. Med., 42:710-3, 1949.

12. SANTA ROSA. C. A. et al. Nuve anos de leptospirose no Instituto Biológico de São Paulo, Rev. Inst. Adolfo Lutz, 29/30:19-27, 1969/70.

13 SAVINO, E. \& RENNELLA, E. Estudios sobre leptospiras. III - Presencia de leptospiras en los perros de la ciudad de Buenos Aires, Rev. Inst. Bact. Malbrán, 12:215-26, 1944.

14. STUART, R. D. Leptospirosis in dogs and other animals. Canad. J. comp. Med., $16: 257-9,1952$.

15. SULZER, C. R. \& JONES, W. L. Leptospirosis methods in laboratory diagnosis. Atlanta, Ga., Department of Health, Education, and Welfare, s. d.

16. SZYFRES, S. B. La leptospirosis como problema de salud humana $y$ animal en America Latina $y$ el area del Caribe. In: Reunion Interamericana sobre el Control de la Fiebre Aftosa y Otras Zoonosis, 8a., Guatemala, 1975. Washington, Organizacion Pananericana de la Salud, 1976. F. 125-41 (OPAS - Publ. cient., 316).

17. VERONESI, Q. et al. Leptospiroses em cães da cidade de São Paulo. Inquérito sorológico. Rev. Inst. Adolpho Lutz, 16 :78-84, 1956.

Recebido para publicação em 08/07/1986 Aprovado para publicaşāo em 12/09/1980 